\title{
Assessment of the Access AMH assay as an automated, high-performance replacement for the AMH Generation II manual ELISA
}

\author{
Kylie Pearson ${ }^{1 *}$ D, Matthew Long ${ }^{2}$, Josephine Prasad ${ }^{2}$, Ye Ying Wu ${ }^{3}$ and Michael Bonifacio ${ }^{2}$
}

\begin{abstract}
Background: The manual Generation II (Gen II) ELISA method used to measure Anti-Müllerian Hormone (AMH) from Beckman Coulter has recently been superseded by a fully automated AMH immunoassay. The aim of this study was to evaluate the performance of the Access AMH assay and directly compare it to the modified Gen II ELISA method. A secondary aim was to verify that the fertile age-related AMH range previously established using the Gen II ELISA could be used to interpret results from the new automated Access assay.
\end{abstract}

Methods: The precision, stability, linearity, measurement range and detection limits were determined using recombinant $\mathrm{AMH}$ and patient serum samples. Different diluents and their effects on $\mathrm{AMH}$ concentration were compared. A correlation study was performed on patient samples to compare the Access AMH assay to the ELISA method on the Access 2 and Dxl800 analysers. The fertile AMH range was verified by comparing the 10th, 50th and 90th percentile values from both methods obtained from 489 natural conception pregnant women.

Results: The Access AMH assay showed good performance across the measuring range for both intra-assay (CV 1.41-3.30\%) and inter-assay (CV 3.04-5.76 \%) precision and acceptable sample stability. Dilution of the high concentration samples with the recommended diluent resulted in a small but significant downward shift in values. The assay was linear over the range of values recommended by the manufacturer, allowing for accurate reporting within the reported range. The two assay types were highly correlated $\left(R^{2}=0.9822\right.$ and 0.9832 for Access 2 and Dxl800, respectively), and the differences observed between the Access2 and DxI800 analysers were within clinically acceptable ranges, indicating that the methods are interchangeable. Furthermore, we demonstrated that results from the published reference range for the Gen II ELISA correlate with those from the automated Access AMH assay.

Conclusion: Here, we verified the published performance of the Access AMH assay and showed excellent correlation with the Gen II ELISA method. Moreover, we validated this correlation by confirming that the results from a fertile AMH reference range established using the preceding Gen II ELISA are interchangeable with the new automated Access AMH assay.

Keywords: Anti-Müllerian Hormone, AMH, Assay, Immunoassay, ELISA, Beckman Coulter, Automated, Reference range

\footnotetext{
* Correspondence: kylie.pearson@genea.com.au

${ }^{1}$ Genea-Canberra, 17B/2 King Street, Deakin ACT 2600, Australia

Full list of author information is available at the end of the article
} 


\section{Background}

Anti-Müllerian hormone $(\mathrm{AMH})$ is expressed by the ovarian granulosa cells of the female ovary where it has important autocrine and paracrine regulator functions in follicle development. It is predominantly produced by the pre-antral and small antral follicles, and production then declines during the final maturation process and luteal phase [1]. AMH acts as an inhibitor of further follicle recruitment and inhibits the response of larger follicles to follicle-stimulating hormone (FSH)-induced growth and selection $[2,3]$.

The number of growing follicles and the resultant level of $\mathrm{AMH}$ released into the circulation is proportionate to the size of the primordial follicle stock [4, 5]. While other tests for ovarian reserve such as basal FSH remain valuable to fertility investigation particularly for patients with reduced ovarian reserve $[6,7]$, the serum concentration of $\mathrm{AMH}$ is gonadotropin independent, thus it remains relatively constant throughout the menstrual cycle $[8,9]$. Consequently, AMH serum concentration has emerged as a unique biological marker for the size of the residual follicular pool that exhibits high correlation with ovarian reserve [10]. This single blood test can additionally assist in the prediction of ovarian response to stimulation, aid in the diagnosis of polycystic ovary syndrome (PCOS) and predict premature ovarian failure, among other uses [11-14].

A number of AMH immunoassays have been developed in the past; however, a lack of standardisation and technical issues between different methods has led to confusion in the interpretation of results and scepticism of AMH test reliability [15-17]. We recently established an AMH reference range of fertile women using the widely-used modified AMH Gen II enzyme-linked immunosorbent assay (ELISA) from Beckman Coulter [18]. The antibodies used in the Gen II assay have now been adopted in the first commercial fully automated AMH assay systems from Roche Diagnostics (Elecsys) and Beckman Coulter (Access) for both the Access2 and DxI800 instruments [19, 20]. Studies have revealed good correlations between the Gen II and Elecsys assays; however, a consensus on correlation between the Gen II ELISA and the new Access AMH assay has not been reached [21, 22].

In the present study, we assessed the accuracy and reproducibility of the Access AMH assay from Beckman Coulter and performed a correlation study with the previous Gen II ELISA method. Furthermore, we determined whether our published age reference range of $\mathrm{AMH}$ values from fertile women could be used to interpret results from the new AMH assay.

\section{Methods}

The Beckman Coulter Access AMH immunoassay was assessed for use on both the Beckman Coulter Access2 and DxI800 analysers. Assay precision was evaluated for both intra- and inter-run precision using $\mathrm{AMH}$ quality control (QC) material consisting of human recombinant $\mathrm{AMH}$ (Beckman Coulter) at three known concentrations. Aliquots of samples were frozen and thawed once prior to testing. Intra-assay performance of the Access $\mathrm{AMH}$ assay was determined from 10 replicates of the assay QC material during the same running cycle, and inter-assay performance was determined by analysing the first run of QC material each day for nine consecutive days. The data was calculated as CV \% (standard deviation/mean $\times 100)$.

Sample stability was assessed on patient sera and QC material. For patient sera, blood was allowed to clot in SST tubes (Becton Dickinson), and the serum was separated by centrifugation according to the manufacturer's recommendations before being stored at $-20{ }^{\circ} \mathrm{C}$ until further analysis. Stability at $4{ }^{\circ} \mathrm{C}$ was determined by testing a sample from one patient in triplicate for eight consecutive days. Stability over freeze/thaw cycles was assessed by testing a fresh sample on day one, then freezing the sample at $-20{ }^{\circ} \mathrm{C}$ and thawing prior to testing for seven cycles. The data was calculated as \% deviation of mean (mean - expected mean/expected mean $\times 100$ ).

Assay linearity was confirmed across the measuring range by testing dilutions of calibrator material (recombinant human AMH; Beckman Coulter) and patient sera. The S5 AMH calibrator was diluted out in a series using Sample Diluent A. The patient sera dilution series was performed using a mix of sera from patients with known high and low $(<0.3 \mathrm{pmol} /$ L) $\mathrm{AMH}$ concentrations to ensure that no matrix effect was present. Linearity was determined using the Cusum test for linearity.

Assay detection limits were determined using doubling dilutions of a patient serum sample with wash buffer (Beckman Coulter). The Limit of Blank (LoB) was calculated as the mean of the blank plus 1.645 times the standard deviation of the blank, while the Limit of Detection (LoD) was calculated as the LoB plus 1.645 times the standard deviation of low-level samples using the highest standard deviation value of the low-level samples tested.

Dilutions of three patient sera samples (AMH concentrations 75, 115 and $140 \mathrm{pmol} / \mathrm{L}$; samples 1,2 and 3 respectively) were tested in duplicate, comparing neat values with 1:5 dilutions in low concentration patient sera, the recommended diluent from Beckman Coulter (Sample Diluent A), or wash buffer. Dilution testing was also performed on patient sera of mid- and highrange concentrations (77 and $177 \mathrm{pmol} / \mathrm{L}$ ), comparing neat values and samples diluted 1:5, 1:10 and 1:16 in Sample Diluent A or patient sera of AMH concentration $<0.1 \mathrm{pmol} / \mathrm{L}$. A set of patient samples of $\mathrm{AMH}$ 
concentration $>70 \mathrm{pmol} / \mathrm{L}(n=27)$ were assayed neat or diluted 1:5 in Sample Diluent A. The results were analysed by a two tailed $t$-test.

The correlation between the new automated Access $\mathrm{AMH}$ assay and the previous Beckman Coulter AMH Gen II ELISA: revised protocol [23] was determined on both the Access2 and DxI800 analysers. Sample AMH concentrations were determined by single measurements from 142 fresh patient serum samples by the Gen II ELISA. Then, the samples were stored at $-20{ }^{\circ} \mathrm{C}$ and thawed once before measurement using the automated Access AMH method for each analyser. A further correlation of 46 samples stored at $-20{ }^{\circ} \mathrm{C}$ was conducted between two separate Access 2 analysers. Regression analysis was performed using Passing-Bablok and BlandAltman methods of comparison [24, 25].

The fertile AMH reference range was previously determined [18]. Briefly, a prospective observational study was conducted on 492 pregnant women in their first trimester, aged between 20 and 44 years, who had all conceived spontaneously without the use of ovarian stimulation drugs within 2-3 months of attempted conception. Blood samples were taken and stored at $-80{ }^{\circ} \mathrm{C}$, and their $\mathrm{AMH}$ concentration was determined using the revised Gen II assay. To test for correlations between the reference ranges determined using the ELISA with the automated AMH assay, 489 of the original samples stored at $-80{ }^{\circ} \mathrm{C}$ were thawed and tested on the DxI800 analyser using the automated AMH assay. The results were analysed to create an age-stratified collection of patient reference ranges. Patients were broken down into 5-year age brackets, and the 10th, 50th and 90th percentile AMH values of each bracket were calculated. These values were plotted using the median age of the patients for each bracket to create a polynomial curve for the limits of the AMH reference range.

Statistical calculations were carried out using Microsoft Excel 14.0 and SPSS Statistics 22.0 for the $t$-tests. Comparison studies were performed using Medcalc Version 15.11.4. All research was conducted in accordance with the ethics guidelines approved by the Genea
Ethics Committee (EC00289) under approval GEC0028 with informed consent obtained from all participants.

\section{Results \\ Precision}

The Access assay showed good performance across the measuring range for the Access AMH Assay on both the Access2 and DxI800 instruments (Table 1). Intra-assay and inter-assay precision ranged from $\mathrm{CV}$ 1.41- $3.30 \%$ and CV 3.04-5.76 \%, respectively, and are within the ranges reported by Beckman Coulter.

\section{Sample stability}

Human recombinant QC material and patient sera stored at $4{ }^{\circ} \mathrm{C}$ were shown to be stable over this time period (deviation from day 1 mean: $-0.67-3.58 \%$ ). The effect of up to five freeze/thaw cycles on sample stability was also minimal (deviation from day 1 mean: -0.11$3.80 \%$; Table 2).

\section{Linearity}

The automated Access assay was established to be remarkably linear over the range of measurement values specified by Beckman Coulter when using either human recombinant AMH material or patient sera (Fig. 1a and b). Linear regression analysis yielded $\mathrm{R}^{2}$ values between 0.9947 and 0.9996 and slope values between 0.9881 and 1.0528. Furthermore, there was no significant deviation from linearity using the Cusum test for linearity under all tested conditions. Similar results were obtained using a dilution series of patient sera diluted in Sample Diluent A (unpublished observations). These data indicate that the linear relationship is maintained under the required assay conditions and thus allows for accurate reporting within the reported range.

\section{Assay limits}

A dilution series of samples at the lower end of the measuring range resulted in good linearity on both the Access2 $\left(R^{2}=0.9995\right.$, slope $\left.=0.9488\right)$ and DxI800 analysers $\left(R^{2}=0.9932\right.$, slope $\left.=0.9093\right)$ (Fig. 1c). The LoD was

Table 1 AMH intra- and inter-assay precision on Access2 and Dxl800 analysers

\begin{tabular}{|c|c|c|c|c|c|c|c|}
\hline & & \multicolumn{3}{|c|}{ Intra-assay precision } & \multicolumn{3}{|c|}{ Inter-assay precision } \\
\hline & & Mean, pmol/L & $\mathrm{SD}, \mathrm{pmol} / \mathrm{L}$ & $C V, \%$ & Mean, pmol/L & $\mathrm{SD}, \mathrm{pmol} / \mathrm{L}$ & $C V, \%$ \\
\hline \multirow[t]{3}{*}{ Access2 } & Control 1 & 6.27 & 0.09 & 1.51 & 6.23 & 0.24 & 3.85 \\
\hline & Control 2 & 31.78 & 0.45 & 1.41 & 31.69 & 0.96 & 3.04 \\
\hline & Control 3 & 97.85 & 1.64 & 1.68 & 97.19 & 3.35 & 3.45 \\
\hline \multirow[t]{3}{*}{ Dxl800 } & Control 1 & 6.40 & 0.21 & 3.30 & 6.31 & 0.28 & 4.36 \\
\hline & Control 2 & 33.36 & 0.68 & 2.04 & 32.49 & 1.87 & 5.76 \\
\hline & Control 3 & 103.18 & 2.98 & 2.89 & 98.54 & 4.52 & 4.59 \\
\hline
\end{tabular}


Table 2 AMH stability under different storage conditions

\begin{tabular}{|c|c|c|c|c|c|c|c|}
\hline & Stored at $4{ }^{\circ} \mathrm{C}$ & & & & Freeze/thaw & & \\
\hline & Mean, pmol/L & $\mathrm{SD}, \mathrm{pmol} / \mathrm{L}$ & Deviation, \% & & Mean, pmol/L & SD, pmol/L & Deviation, \% \\
\hline Day 1 & 5.96 & 0.16 & 0.00 & Fresh & 5.96 & 0.16 & 0.00 \\
\hline Day 2 & 5.83 & 0.09 & -2.29 & $\times 1$ & 5.96 & 0.08 & -0.11 \\
\hline Day 3 & 5.77 & 0.03 & -3.24 & $\times 2$ & 5.87 & 0.04 & -1.57 \\
\hline Day 4 & 5.92 & 0.04 & -0.67 & $\times 3$ & 5.91 & 0.14 & -0.89 \\
\hline Day 5 & 5.75 & 0.18 & -3.58 & $x 4$ & 5.74 & 0.09 & -3.80 \\
\hline Day 6 & 6.09 & 0.05 & 2.07 & $\times 5$ & 5.81 & 0.18 & -2.63 \\
\hline Day 7 & 5.75 & 0.01 & -3.52 & $x 6$ & 5.74 & 0.04 & -3.75 \\
\hline Day 8 & 5.83 & 0.22 & -2.24 & $x 7$ & 5.78 & 0.07 & -3.13 \\
\hline
\end{tabular}

A

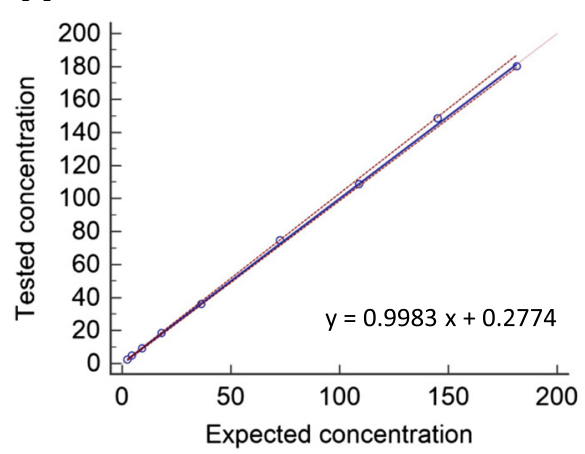

B

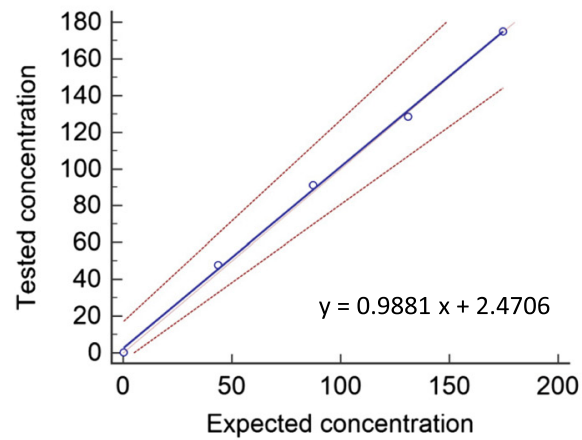

C

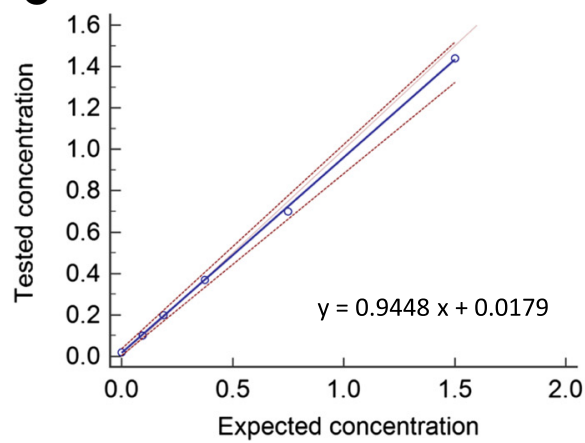

DXI800
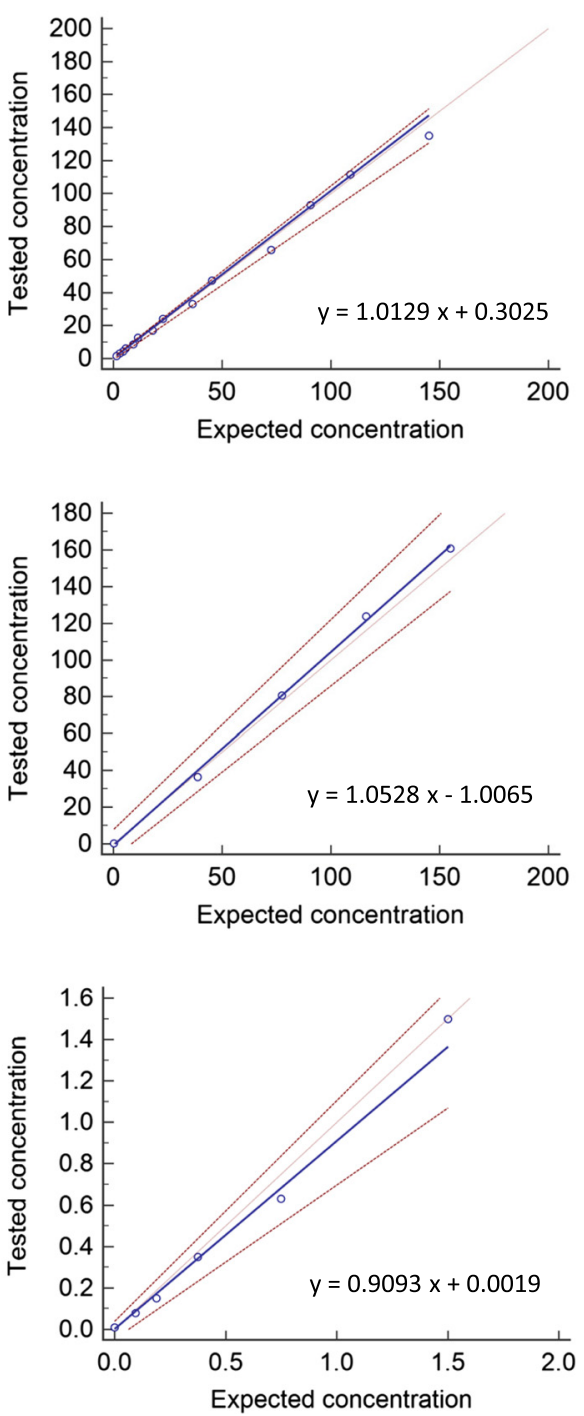

Fig. 1 Linearity of the Access AMH assay on the Access2 and Dxl800 analysers using a. calibrator material or b. patient sera. c. Linearity at the low end of the concentration range using patient sera. Regression line (blue), $95 \%$ confidence intervals (red). All results are shown in pmol/L 
calculated to be 0.1055 on the Access 2 and 0.0994 on the DxI800, while the LoB was calculated to be 0.0200 on the Access 2 and 0.0352 on the DxI800. All determined assay limits were lower than the values designated by Beckman Coulter $(\mathrm{LoD}=0.14 \mathrm{pmol} / \mathrm{L}, \mathrm{LoB}=$ $0.07 \mathrm{pmol} / \mathrm{L}$ ) and confirmed the high sensitivity of this new assay.

\section{Dilution testing}

A comparison of neat AMH concentrations with those obtained using three different dilution materials revealed that samples diluted in patient sera of low concentration showed the least deviation from the neat value, followed by Sample Diluent A and wash buffer (Additional file 1: Figure S1a). The dilution effect was further investigated by performing 1:5, 1:10 and 1:16 dilutions (1:16 being the recommended dilution factor from Beckman Coulter) on samples from patients with mid-range and high-range $\mathrm{AMH}$ concentrations and measuring $\mathrm{AMH}$ using the automated Access assay (Table 3). Dilution in low AMH concentration sera resulted in -2.56-5.21\% deviation from the mean neat result, while dilution in Sample Diluent A resulted in a deviation from the neat value of $-3.63-13.31 \%$. This finding was confirmed by testing a cohort of patient samples of AMH concentration $>70 \mathrm{pmol} / \mathrm{L}$ and comparing the neat results with the diluted (1:5 in Sample Diluent A) results (Additional file 1: Figure S1b). Overall, the results display a consistent decrease of approximately $10 \%$ upon sample dilution, which, while significant $(t$-test $p<0.0001)$, would not be clinically relevant. These data suggest that there is no need for dilution of samples that are $>70 \mathrm{pmol} / \mathrm{L}$, as required for the previous Gen II ELISA; in fact, the opposite effect occurs upon dilution, where sample concentrations are negatively biased.

\section{Correlation studies between assays and analysers}

The correlation of the new Access AMH assay with the preceding revised AMH Gen II ELISA was determined by a comparative study of 142 patient samples (Fig. 2a).
Passing-Bablok regression analysis comparing the Gen II ELISA with the Access AMH assay run on the Access2 and DxI800 analysers resulted in $R^{2}$ values of 0.9822 and 0.9832 and slope values of 1.0014 and 0.9231 , respectively. All regression curves showed no significant deviation from linearity, indicating good correlation between these methods. The Bland-Altman analyses indicate an absence of bias across the analytical range for all comparisons.

The same patient cohort was used to perform a correlation study between the Access2 and DxI800 analysers using the Access AMH assay $\left(R^{2}=0.9964\right.$, slope $=$ 1.0758). A smaller cohort of 46 patient samples was used to perform a comparison between two Access 2 analysers located in different geographical locations $\left(R^{2}=0.9820\right.$, slope $=1.0317)$. Regression analysis and Bland-Altman plots demonstrate high correlation across the measuring range between the results obtained on the different instruments (Fig. 2b).

\section{Correlation study of normal AMH reference ranges}

A large study cohort of 489 pregnant patients who were used previously to establish a fertile $\mathrm{AMH}$ reference range using the Gen II ELISA were re-tested using the Access AMH assay. The results from both assays were analysed to create an age-stratified collection of patient reference ranges by plotting the age group against the values of the median, 10th, 50th and 90th percentiles (Fig. 3). The results between the two methods were highly correlated (average CV $6.7 \%$ ), and the same significant curvilinear relationship between $\mathrm{AMH}$ and age was confirmed using the new AMH test. As a result, we conclude that the previously determined reference range can be used to interpret data from either assay.

\section{Discussion}

AMH measurement is emerging as an extremely useful tool in a number of areas of reproductive medicine. However, technical issues with regards to stability and lack of standardisation between past AMH assays have

Table 3 Comparison of different dilution methods using the Access AMH Assay

\begin{tabular}{|c|c|c|c|c|c|c|c|}
\hline & \multirow[b]{2}{*}{ Dilution } & \multicolumn{3}{|c|}{ Dilution in patient sera } & \multicolumn{3}{|c|}{ Dilution in sample diluent A } \\
\hline & & Mean, pmol/L & $C V, \%$ & Deviation, \% & Mean, pmol/L & $C V, \%$ & Deviation, \% \\
\hline \multirow[t]{4}{*}{ Sample 1} & Neat & 76.90 & 0.50 & 0.00 & 76.90 & 0.50 & 0.00 \\
\hline & $1: 5$ & 74.82 & 1.09 & -2.71 & 68.90 & 1.73 & -10.41 \\
\hline & $1: 10$ & 74.63 & 1.59 & -2.96 & 66.28 & 2.74 & -13.81 \\
\hline & $1: 16$ & 77.81 & 1.11 & 1.18 & 68.04 & 1.36 & -11.52 \\
\hline \multirow[t]{4}{*}{ Sample 2} & Neat & 170.64 & 1.83 & 0.00 & 170.64 & 1.83 & 0.00 \\
\hline & $1: 5$ & 177.69 & 1.10 & 4.13 & 164.44 & 1.24 & -3.63 \\
\hline & $1: 10$ & 176.71 & 0.64 & 3.56 & 154.80 & 0.46 & -9.28 \\
\hline & $1: 16$ & 179.53 & 3.55 & 5.21 & 153.65 & 3.07 & -9.96 \\
\hline
\end{tabular}



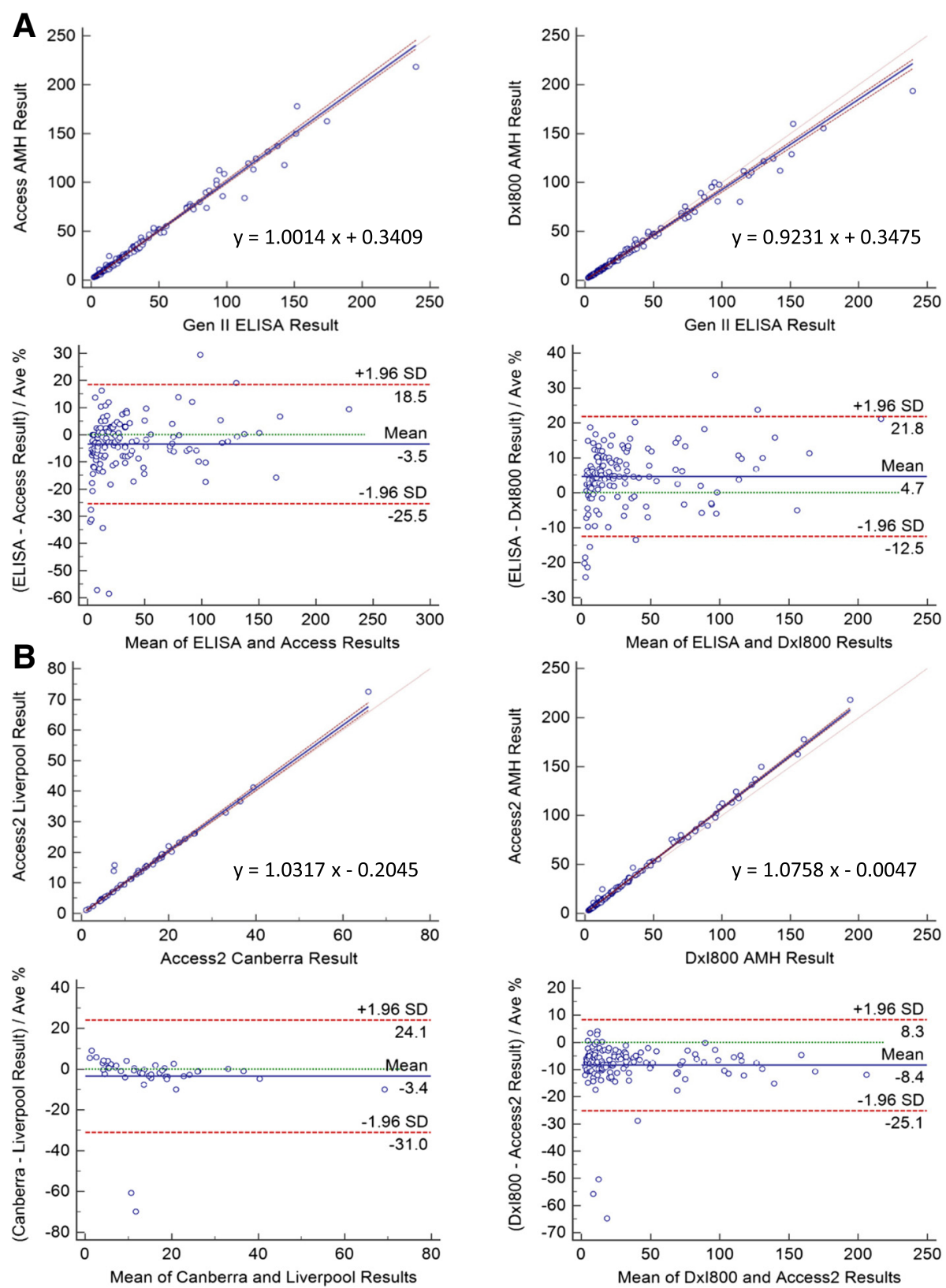

Fig. 2 a. Correlation between Access AMH and Gen II ELISAs on the Access2 and DxI800 instruments $(n=142)$. b. Correlation between results from Access2 analysers in different locations $(n=46)$ and Access2 and Dxl800 analysers $(n=142)$. Upper panels represent the Passing-Bablok diagram with the regression line (blue) and the $95 \%$ confidence interval (red). Lower panels represent the Bland-Altman plots with mean (blue) and $95 \%$ confidence intervals (red). All results are shown in $\mathrm{pmol} / \mathrm{L}$

led to uncertainty in AMH test reliability and result interpretation $[15,26,27]$. The findings of the correlation studies herein demonstrate agreement between the results generated by the AMH Gen II ELISA and the Access AMH Assay. Comparing the automated Access and manual ELISA method using the Access2 analyser showed near complete agreement, while results from the DxI800 analyser showed a slight difference within acceptable clinical ranges. These findings indicate that the past issues have been overcome when comparing the new and previous Beckman Coulter methods.

In support of our results, van Helden and Weiskirchen [22] demonstrated good correlations between the Gen II ELISA and both the Access AMH assay and the new automated Elecsys system from Roche, both of which utilise the same monoclonal antibodies. The authors also demonstrated an extremely tight correlation between the Access and Elecsys assays, and these results were further 


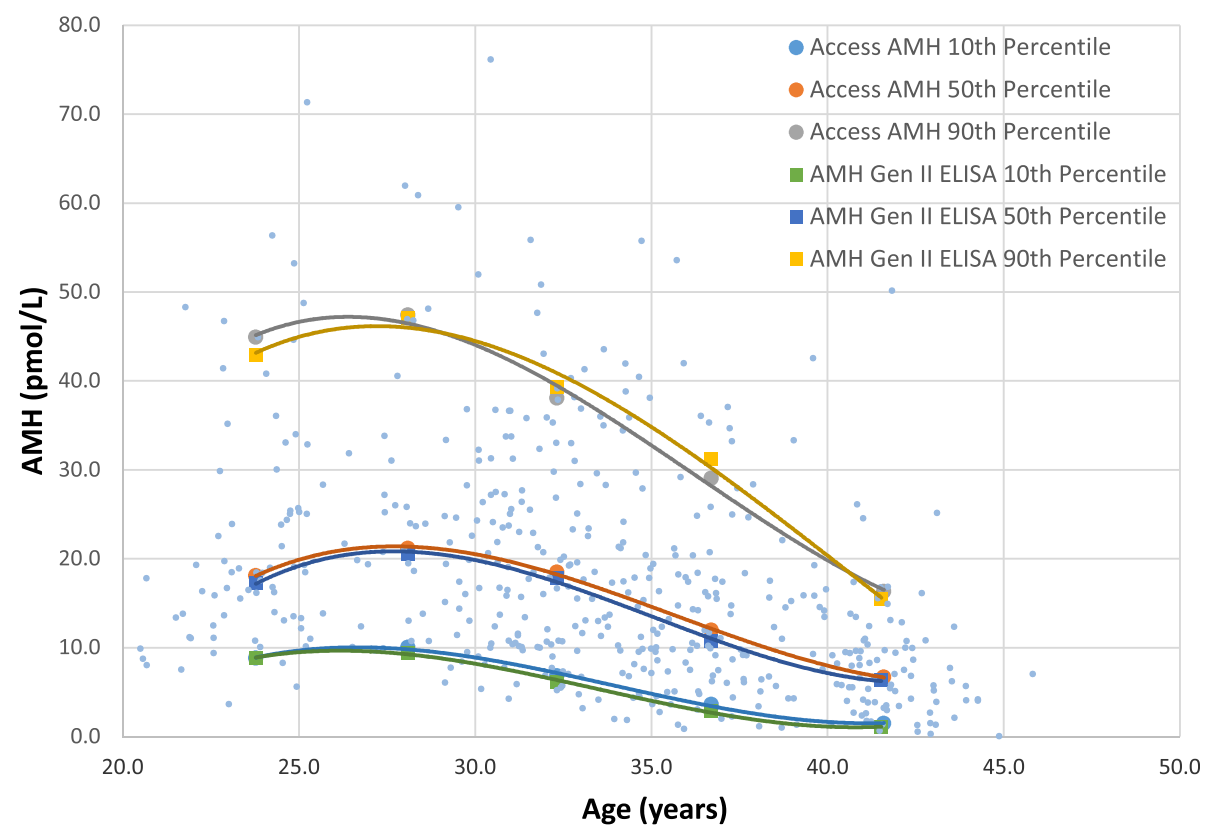

Fig. 3 Correlation between automated AMH and Gen II ELISA normal female reference ranges. Individual results (blue points) and 10th, 50th and 90th percentile median values for 5 -year age groups for Access AMH and Gen II ELISA methods $(n=489)$

validated by Nelson et al. [21]. Interestingly, Nelson et al. [21] report a $22 \%$ decrease in expected values and distinct systematic bias between the old and new Beckman Coulter AMH assays with increasing concentrations. Different sample storage and handling conditions may have contributed to this discordance. Hyldgaard et al. [28] demonstrated a similar pattern of bias between the Gen II and Elecsys methods and proposed that this may be in part due to inter-laboratory bias with the Gen II method. The Gen II ELISA is a manual method and thus would be more prone to inter-operator bias and variation from a number of sources within the assay itself. Our ELISAs were performed by a single experienced operator and we used a large sample size, which may account for the close correlation observed by our laboratory. Furthermore, we have shown that the dilution of high concentration samples and the use of different diluents can cause a shift in values, which may explain the conflicting results with high concentration samples.

Assessment of the Access AMH assay revealed excellent linearity and good performance across the measuring range for both intra-assay and inter-assay precision as would be expected for an automated immunoassay. This assay exhibited greatly increased sensitivity when compared to previous manual methods and aligned with literature from the manufacturer allowing for accurate reporting to $0.1 \mathrm{pmol} / \mathrm{L}$. We demonstrated high levels of AMH immunoreactive stability under refrigerated and freeze/thaw conditions, though the long-term effect of storage under different conditions was not within the scope of this study. The results of the dilution testing revealed that $\mathrm{AMH}$ samples greater than $70 \mathrm{pmol} / \mathrm{L}$ do not need to be diluted as was required with the previous Gen II ELISA. In fact, our study revealed that dilution caused a negative shift of approximately $10 \%$, indicating care should be applied in the interpretation of results from diluted samples. This shift would not, however, have a significant effect on clinical outcome.

This is the first paper to report an established fertile age-related $\mathrm{AMH}$ reference range that is compatible with the automated Access AMH assay. Numerous studies have determined AMH reference ranges; however, the majority of this research was conducted using infertile or presumably fertile study groups and former methods [15, 16, 29-33]. Bonifacio et al. [18] recently published an AMH normogram using the revised Gen II ELISA method on a large cohort of first trimester pregnant patients who had conceived by natural and unaided means. AMH levels have been shown by a number of studies to exhibit little variation within and between menstrual cycles and to be stable from pre-pregnancy through the first trimester of pregnancy [34-36]; therefore, the study group was considered as representative of a fertile population. Here, we conducted a full study comprised of the same cohort using the automated Access AMH assay and the Gen II ELISA. A comparison of the results between the two assays showed variation within the performance limits of both analysis methods. This study further validated the results of our method 
comparison and confirmed that our fertile reference range of AMH values can be applied to both the previous ELISA and the new automated assay from Beckman Coulter.

\section{Conclusions}

The two most important influences on the acquisition of reliable clinical information are the dependability of the measurements and the interpretation of the results. This study verified the published performance of the new automated Access AMH assay from Beckman Coulter showing a measuring range adequate for most IVF applications and the ability to detect values consistently across this range. The automated assay exhibited high levels of stability and sensitivity and showed correlation with the existing ELISA method and between analyser platforms. Furthermore, an age-related reference range of $\mathrm{AMH}$ values was established for patients not already undergoing infertility assessment, allowing for accurate extrapolation of data to tailor treatment and prognosis prediction in the wider population. These findings are an encouraging step towards the necessary establishment of universal clinically relevant cut-off values and the standardisation of $\mathrm{AMH}$ assay results.

\section{Additional file}

Additional file 1: Figure S1. a. Effect of different diluents on the expected outcome of three patient samples. b. Effect of 1:5 dilution with Sample Diluent A on patient AMH concentration compared to neat values using the Access AMH assay. (PDF $25 \mathrm{~kb}$ )

\section{Abbreviations}

AMH: Anti-Müllerian hormone; ELISA: Enzyme-linked immunosorbent assay; FSH: Follicle-stimulating hormone; Gen II: Generation II; IVF: In vitro fertilisation; LoB: limit of blank; LoD: limit of detection; PCOS: Polycystic ovary syndrome; QC: quality control material.

\section{Competing interests}

The authors declare that they have no competing interests.

\section{Authors' contributions}

$\mathrm{KP}, \mathrm{ML}$ and $\mathrm{MB}$ conceived of all of the experiments, and KP, JP and YW carried out the experiments. KP, ML and MB carried out the analysis and interpretation of the data. KP prepared the manuscript, and KP, ML and MB critically revised the manuscript. All authors read and approved the final manuscript version.

\section{Acknowledgements}

We would like to thank Sue Khouri and YoonJoo Choi for their contribution to the analysis of the AMH samples. Thank you also to Genevieve Maybury, Neha Pal, Mel Bauer and Emily Carlin for their project support. We thank American Journal Experts for English language editing and to Christine Napier and Cara Bradley for their help with the analysis and critical revision of the manuscript.

\section{Author details}

${ }^{1}$ Genea-Canberra, 17B/2 King Street, Deakin ACT 2600, Australia. ${ }^{2}$ Genea-Sydney City, Level 3/321 Kent Street, Sydney, NSW 2000, Australia. ${ }^{3}$ Genea-South West, 173-175 Bigge Street, Liverpool, NSW 2170, Australia.
Received: 28 December 2015 Accepted: 8 February 2016

Published online: 16 February 2016

\section{References}

1. Sowers MR, Eyvazzadeh AD, McConnell D, Yosef M, Jannausch ML, Zhang D, et al. Anti-mullerian hormone and inhibin B in the definition of ovarian aging and the menopause transition. J Clin Endocrinol Metab. 2008;93(9): 3478-83.

2. Durlinger AL, Visser JA, Themmen AP. Regulation of ovarian function: the role of anti Mullerian hormone. Reproduction. 2002;124:601-9.

3. Teixeira J, Maheswaran S, Donahoe PK. Mullerian inhibiting substance: an instructive developmental hormone with diagnostic and possible therapeutic applications. Endocr Rev. 2001;22:657-74.

4. Fanchin R, Schonauer LM, Righini C, Guibourdenche J, Frydman R, Taieb J. Serum anti-Mullerian hormone is more strongly related to ovarian follicular status than serum inhibin B, estradiol, FSH and LH on day 3. Hum Reprod. 2003:18:323-7.

5. van Rooij IA, Baroekmans FJ, Scheffer GJ, Looman CW, Habbema JD, De Jong $\mathrm{FH}$, et al. Serum anti-Mullerian hormone levels best reflect the reproductive decline with age in normal women with proven fertility: a longitudinal study. Fertil Steril. 2005;83:979-87.

6. Gleicher N, Weghofer A, Barad DH. Discordances between follicle stimulating hormone (FSH) and anti-Müllerian hormone (AMH) in female infertility. Reprod Biol Endocrinol. 2010. doi:10.1186/1477-7827-8-64.

7. Hussain M, Cahill D, Akande V, Gordon U. Discrepancies between antimullerian hormone and follicle stimulating hormone in assisted reproduction. Obst Gynecol Int. 2013. doi:10.1155/2013/383278.

8. Tsepelidis S, Devreker F, Demeestere I, Flahaut A, Gervy C, Englert Y. Stable serum levels of anti-Mullerian hormone during the menstrual cycle: a prospective study in normo-ovulatory women. Hum Reprod. 2007;22(7):1837-40.

9. Hehenkamp WJ, Looman CW, Themmen AP, de Jong FH, Te Velde ER, Broekmans FJ. Anti-Mullerian hormone levels in the spontaneous menstrual cycle do not show substantial fluctuation. J Clin Endocrinol Metab. 2006; 91(10):4057-63.

10. Niodromiti S, Anderson RA, Nelson SM. Technical and performance characteristics of anti-Mullerian hormone and antral follicle count as biomarkers of ovarian response. Hum Reprod Update. 2014;21(6):698-710.

11. Broer SL, Dolleman M, Opmeer BC, Fauser BC, Mol BW, Broekmans FJ. AMH and AFC as predictors of excessive response in controlled ovarian hyperstimulation: a meta-analysis. Hum Reprod Update. 2011;17(1):46-54.

12. Elgindy EA, El-Haieg DO, El-Sebaey A. Anti-Mullerian hormone: correlation of early follicular, ovulatory and midluteal levels with ovarian response and cycle outcome in intracytoplasmatic sperm injection patients. Fertil Steril. 2008;89:1670-6.

13. Hazout A, Bouchard P, Seifer DB, Aussage $P$, Junca AM, Cohen-Bacrie $P$. Serum antimullerian hormone/mullerian inhibiting substance appears to be a more discriminatory marker of assisted reproductive technology outcome than follicle-stimulating hormone, inhibin B, or estradiol. Fertil Steril. 2004; 82:1323-9.

14. Dewailly D, Andersen CY, Balen A, Broekmans F, Dilaver N, Fanchin R, et al. The physiology and clinical utility of anti-Mullerian hormone in women. Hum Reprod Update. 2014;20:370-85.

15. Han X, McShane M, Sahertian R, White C, Ledger W. Pre-mixing serum samples with assay buffer is a prerequisite for reproducible anti-Mullerian hormone measurement using the Beckman Coulter Gen II assay. Hum Reprod. 2014;29:1042-8.

16. Nelson SM, Iliodromiti S, Fleming R, Anderson R, McConnachie A, Messow $\mathrm{CM}$. Reference range for the AMH Generation II assay: a population study of 10,984 women, with comparison to the established DSL nomogram. Fertil Steril. 2014;101:413-9.

17. Pankhurst MW, Chong YH, McLennan IS. Enzyme-linked immunosorbent assay measurements of antimüllerian hormone (AMH) in human blood are a composite of the uncleaved and bioactive cleaved forms of $\mathrm{AMH}$. Fertil Steril. 2014;101(3):846-50.

18. Bonifacio M, Bradley CK, Karia S, Livingstone M, Bowman MC, McArthur SJ. The original Beckman Coulter Generation II assay significantly underestimates $\mathrm{AMH}$ levels compared with the revised protocol. J Assist Reprod Genet. 2015. doi:10.1007/s10815-015-0579-y.

19. Gassner D, Jung R. First fully automated immunoassay for anti-Mullerian hormone. Clin Chem Lab Med. 2014;52:1143-52. 
20. Groome N. The design features and performance of a state-of-the-art fullyautomated anti-mullerian hormone assay for the Beckman Access Family of immunoassay systems. Beckman Coulter. 2015. http://www.beckmancoulteramh.com/en/wp-content/uploads/Access-AMH-Technical-Bulletin-14-072015.pdf.

21. Nelson SM, Pastuszek E, Kloss G, Malinowska I, Liss J, Lukaszuk A, et al. Two new automated, compared with two enzyme-linked immunosorbent, antimüllerian hormone assays. Fertil Steril. 2015;104(4):1016-21.

22. van Helden J, Weiskirchen R. Performance of the two new fully automated anti-Müllerian hormone immunoassays compared with the clinical standard assay. Hum Reprod. 2015;30:1918-26.

23. Beckman Coulter. Urgent Field Safety Notice AMH Gen II ELISA (REF A79765). 2013. https://www.swissmedic.ch/recalllists_dl/08039/Vk_ 20121205_01-e3.pdf.

24. Passing $\mathrm{H}$, Bablok W. A new biometrical procedure for testing the equality of measurements from two different analytical methods. J Clin Chem Clin Biochem. 1983;21:709-20.

25. Bland JM, Altman DG. Statistical methods for assessing agreement between two methods of clinical measurements. Lancet. 1986;8:307-10.

26. Iliodromiti S, Nelson SM. Ovarian response biomarkers: physiology and performance. Curr Opin Obstet Gynecol. 2015;27(3):182-6.

27. Li HW, Ng EH, Wong BP, Anderson RA, Ho PC, Yeung WS. Correlation between three assay systems for anti-Müllerian hormone (AMH) determination. J Assist Reprod Genet. 2012;29(12):1443-6.

28. Hyldgaard J, Bor P, Ingerslev HJ, Tørring N. Comparison of two different methods for measuring anti-mullerian hormone in a clinical series. Reprod Biol Endocrinol. 2015. doi:10.1186/s12958-015-0101-5.

29. Masse V, Ferrari P, Boucoiran I, Delotte J, Isnard V, Bongain A. Normal serum concentrations of anti-Mullerian hormone in a population of fertile women in their first trimester of pregnancy. Hum Reprod. 2011;26:3431-6.

30. Tehrani FR, Mansournia MA, Solaymani-Dodaran M, Azizi F. Age-specific serum anti-Mullerian hormone levels: estimates from a large population based sample. Climacteric. 2014;17:591-7.

31. La Marca A, Sighinolfi G, Giulini S, Traglia M, Argento C, Sala C, et al. Normal serum concentrations of anti-Mullerian hormone in women with regular menstrual cycles. Reprod Biomed Online. 2010;21:463-9.

32. Shebl O, Ebner T, Sir A, Schreier-Lechner E, Mayer RB, Tews G, et al. Age related distribution of basal serum $\mathrm{AMH}$ level in women of reproductive age and a presumably healthy cohort. Fertil Steril. 2011:95:832-4.

33. Tremellen K, Zander-Fox D. Serum anti-Mullerian hormone assessment of ovarian reserve and polycystic ovary syndrome status over the reproductive lifespan. Aust N Z J Obstet Gynaecol. 2015;55(4):384-9.

34. Nelson SM, Stewart F, Fleming R, Freeman DJ. Longitudinal assessment of antimullerian hormone during pregnancy-relationship with maternal adiposity, insulin, and adiponectin. Fertil Steril. 2010;93:1356-8.

35. La Marca A, Giulini S, Orvieto R, De Leo V, Volpe A. Anti-Mullerian hormone concentrations in maternal serum during pregnancy. Hum Reprod. 2005:20:1569-72.

36. Lutterodt M, Byskov AG, Skouby SO, Tabor A, Yding AC. Anti-Mullerian hormone in pregnant women in relation to other hormones, fetal sex and in circulation of second trimester fetuses. Reprod Biomed Online. 2009;18:694-9.

\section{Submit your next manuscript to BioMed Central and we will help you at every step:}

- We accept pre-submission inquiries

- Our selector tool helps you to find the most relevant journal

- We provide round the clock customer support

- Convenient online submission

- Thorough peer review

- Inclusion in PubMed and all major indexing services

- Maximum visibility for your research

Submit your manuscript at www.biomedcentral.com/submit

) Biomed Central 